Acta Phys. Hung. A 19/1 (2004) 000-000

HEAVY ION

\title{
Results on hyperon production from the NA57 experiment
}

\author{
Ladislav Šándor for the NA57 collaboration:
}

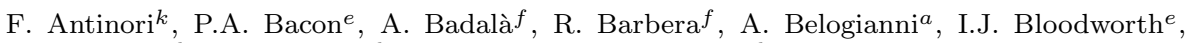
M. Bombara ${ }^{h}$, G.E. Bruno ${ }^{b}$, S.A. Bulle, R. Caliandro ${ }^{b}$, M. Campbell ${ }^{g}$, W. Carena ${ }^{g}$, N. Carrer ${ }^{g}$, R.F. Clarke $^{e}$, A. Dainese ${ }^{k}$, D. Di Bari ${ }^{b}$, S. Di Liberto $^{n}$, R. Divia ${ }^{g}$, D. Elia $^{b}$, D. Evans ${ }^{e}$, G.A. Feofilov ${ }^{p}$, R.A. Fini ${ }^{b}$, P. Ganoti ${ }^{a}$, B. Ghidini ${ }^{b}$, G. Grella ${ }^{o}$, H. Helstrup ${ }^{d}$, K.F. Hetland ${ }^{d}$, A.K. Holme ${ }^{j}$, A. Jacholkowski ${ }^{f}$, G.T. Jones ${ }^{e}$, P. Jovanovic ${ }^{e}$, A. Jusko ${ }^{e}$, R. Kamermans ${ }^{r}$, J.B. Kinson ${ }^{e}$, K. Knudson ${ }^{g}$, V. Kondratiev ${ }^{p}$, I. Králik ${ }^{h}$, A. Kravčáková ${ }^{i}$, P. Kuijer ${ }^{r}$, V. Lenti ${ }^{b}$, R. Lietava ${ }^{e}$, G. Løvhøiden ${ }^{j}$, V. Manzari $^{b}$, M.A. Mazzoni ${ }^{n}$, F. Meddi $^{n}$, A. Michalon ${ }^{q}$, M. Morando ${ }^{k}$, P.I. Norman ${ }^{e}$, A. Palmeri ${ }^{f}$, G.S. Pappalardo ${ }^{f}$, B. Pastirčák ${ }^{h}$, R.J. Platt ${ }^{e}$, E. Quercigh ${ }^{k}$, F. Riggi ${ }^{f}$, D. Röhrich ${ }^{c}$, G. Romano ${ }^{o}$, K. Šafař́k ${ }^{g}$, L. Śándor ${ }^{h}$, E. Schillings ${ }^{r}$, G. Segato ${ }^{k}$, M. Senél ${ }^{2}$ R. Senél ${ }^{l}$ W. Snoeys ${ }^{g}$, F. Soramel ${ }^{k}$, M. SpyropoulouStassinaki $^{a}$, P. Staroba ${ }^{m}$, R. Turrisi ${ }^{k}$, T.S. Tveter ${ }^{j}$, J. Urbán ${ }^{i}$, P. van de $\operatorname{Ven}^{r}$,

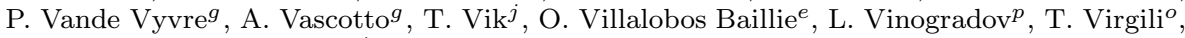
M.F. Votruba ${ }^{e}$, J. Vrláková ${ }^{i}$ and P. Závada ${ }^{m}$

${ }^{a}$ Physics Department, University of Athens, Athens, Greece

${ }^{b}$ Dipartimento I.A. di Fisica dell'Università e del Politecnico di Bari and INFN, Bari, Italy

${ }^{c}$ Fysisk institutt, Universitetet i Bergen, Bergen, Norway

${ }^{d}$ Høgskolen i Bergen, Bergen, Norway

e School of Physics and Astronomy, University of Birmingham, Birmingham, UK

$f$ Dipartimento di Fisica dell'Università and Sezione INFN, Catania, Italy

$g$ CERN, European Laboratory for Particle Physics, Geneva, Switzerland

$h$ Institute of Experimental Physics, Slovak Academy of Science, Košice, Slovakia

${ }^{i}$ P.J. Šafárik University, Košice, Slovakia

$j$ Fysisk institutt, Universitetet i Oslo, Oslo, Norway

${ }^{k}$ Dipartimento di Fisica dell'Università and Sezione INFN, Padua, Italy

${ }^{l}$ Collège de France and IN2P3, Paris, France

$m$ Institute of Physics, Academy of Science of the Czech Republic, Prague, Czech Republic

$n$ Dipartimento di Fisica dell'Università "La Sapienza" and Sezione INFN, Rome,Italy

o Dipartimento di Scienze Fisiche "E.R. Caianiello" dell'Università and INFN, Salerno, Italy

$p$ State University of St. Petersburg, St. Petersburg, Russia

${ }^{q}$ Institut de Recherches Subatomiques, IN2P3/ULP, Strasbourg, France

$r$ Utrecht University and NIKHEF, Utrecht, The Netherlands

* Permanent address: University of Udine, Italy

Received 15 April 2004

Abstract. Recent results on hyperon production in $\mathrm{Pb}-\mathrm{Pb}$ collisions from the NA57 experiment are reported. Strangeness enhancements and the transverse mass spectra properties at $158 \mathrm{GeV}$ per nucleon are described.

Keywords: hyperons, strangeness enhancement, blast-wave model

PACS: 25.75.Dw, 25.75.Ld 


\section{Introduction}

The extensive study of ultrarelativistic heavy-ion collisions in the past two decades was motivated mainly by the QCD prediction that at sufficiently high energy density excited matter undergoes a phase transition to a system of deconfined quarks and gluons (quark-gluon plasma, QGP). Strange particle production has proven to be a powerful tool for the investigation of the dynamics of heavy-ion collisions at high energies (see, e.g. [1]).

In particular, the WA97 experiment at the CERN SPS has measured an enhanced production of strange and multi-strange hyperons in central $\mathrm{Pb}-\mathrm{Pb}$ collisions at $158 \mathrm{~A} \mathrm{GeV} / c$ with respect to proton induced reactions [2]. The observed pattern of the enhancements, increasing with the strangeness content of the particle, was predicted by Rafelski and Müller [3] more than 20 years ago as a consequence of a QGP formation.

NA57, a second generation SPS heavy-ion experiment, continues and extends the study of the production of strange and multi-strange particles in $\mathrm{Pb}-\mathrm{Pb}$ collisions initiated by the WA97 experiment, collecting data in wider centrality range and at two beam momenta - 40 and $158 \mathrm{AGeV} / c$. In this paper, based on a brief review of recent NA57 results given at the 3rd Budapest winter school on heavy-ion physics in December 2003, we concentrate on strangeness enhancement measurements and on the analysis of the transverse mass spectra of hyperons from $\mathrm{Pb}-\mathrm{Pb}$ collisions at $158 \mathrm{~A} \mathrm{GeV/c}$.

\section{The NA57 experiment}

The NA57 set-up and experimental procedure are described elsewhere [4, 5, 6]. A telescope of compactly packed high granularity silicon pixel detectors, located in a $1.4 \mathrm{~T}$ magnetic field, is used as main tracking device. The configuration and geometry of telescope enable to measure particle yields and spectra over half a unit of rapidity around the mid-rapidity. The centrality trigger, based on an array of scintillator counters, selects the $60 \%$ most central fraction of the $\mathrm{Pb}-\mathrm{Pb}$ inelastic cross section. Two stations of silicon microstrip detectors provide data for the measurement of the charged particle multiplicity used for the offline collision centrality determination. As a measure of centrality we use the number of wounded nucleons computed from the measured trigger cross sections via the Glauber model [ 7].

NA57 has collected $\mathrm{Pb}-\mathrm{Pb}$ data samples at both 158 and $40 A \mathrm{GeV} / c$ beam momentum. In order to study the strangeness enhancements at both energies the knowledge of hyperon production in elementary hadron processes is necessary. For this purpose NA57 has also collected a reference data sample of $\mathrm{p}-\mathrm{Be}$ collisions at $40 A \mathrm{GeV} / c$. At $158 A \mathrm{GeV} / c$ the $\mathrm{p}$-Be reference data available from the WA97 experiment were utilized.

The $\mathrm{K}_{S}^{0}$ mesons, the $\Lambda, \Xi^{-}$and $\Omega^{-}$hyperons and their antiparticles are identified by reconstructing their weak decays into final states containing charged particles only. The selection procedure allows us to extract strange particle signals with 
a negligible level of background. Data are corrected for geometrical acceptance and for detector and reconstruction inefficiencies. We have used all the reconstructed multi-strange particles, while for the much more abundant $\mathrm{K}_{S}^{0}, \Lambda$ and $\bar{\Lambda}$ particles we only corrected a fraction of the total data samples in order to reach a statistical accuracy better than the limits imposed by the systematics. The systematic errors for both particle yields and spectra are estimated to be about $10 \%$ for $\Lambda, \Xi$ and $\simeq 15 \%$ for $\Omega$ hyperons.

The double differential cross sections for each particle under study were fitted using the expression

$$
\frac{\mathrm{d}^{2} N}{\mathrm{~d} m_{\mathrm{T}} \mathrm{d} y}=f(y) m_{\mathrm{T}} \exp \left(-\frac{m_{\mathrm{T}}}{T_{a p p}}\right)
$$

where $m_{\mathrm{T}}=\sqrt{m^{2}+p_{\mathrm{T}}^{2}}$ is the transverse mass and $T_{a p p}$ is the inverse slope parameter, assuming the rapidity distribution to be flat in our limited acceptance region $(f(y)=$ const. $)$.

\section{Particle yields and strangeness enhancements}

Using the parametrization given by equation (11), with the $T_{a p p}$ value extracted from from the maximum likelihood fit to the data, one can determine the yield
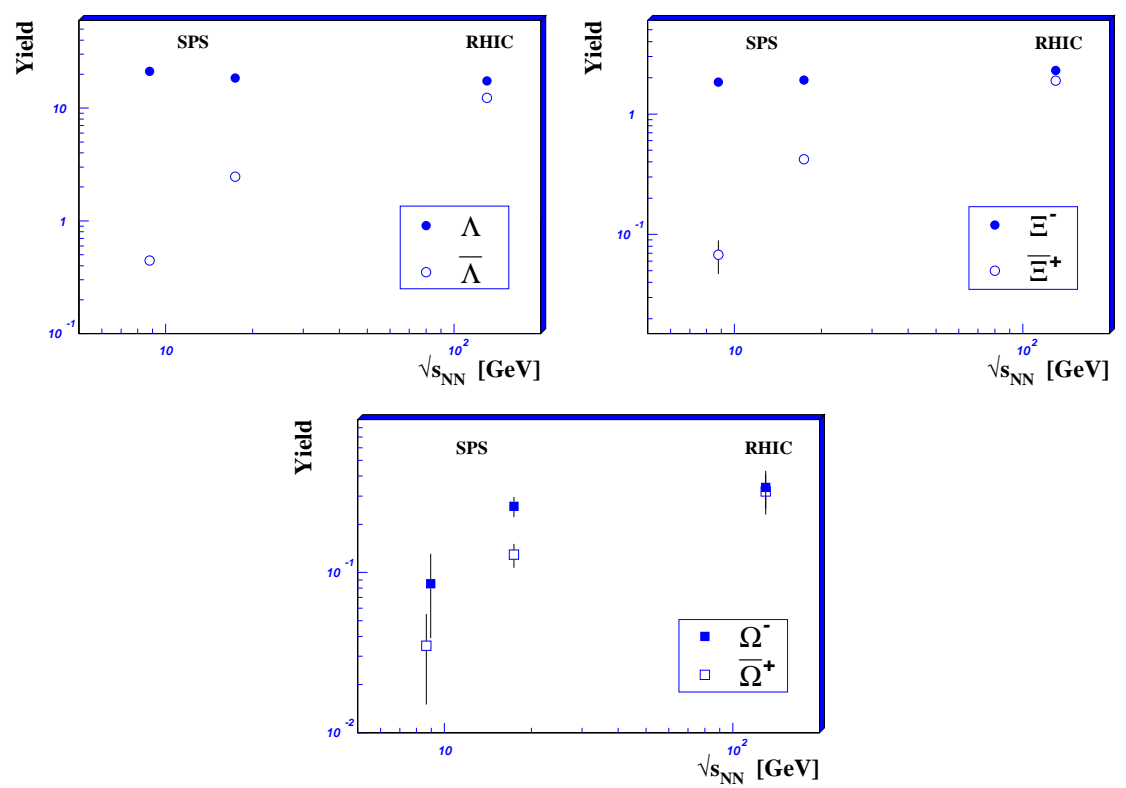

Fig. 1. Energy dependence of hyperon yields $Y$ in central heavy-ion collisions. 
(number of particles per rapidity unit centred at mid-rapidity $y_{0}$ ) of each particle under study:

$$
Y=\int_{m}^{\infty} \mathrm{d} m_{\mathrm{T}} \int_{y_{0}-0.5}^{y_{0}+0.5} \frac{\mathrm{d}^{2} N}{\mathrm{~d} m_{\mathrm{T}} \mathrm{d} y} \mathrm{~d} y
$$

The data have been divided into five centrality classes $(0-4)^{1}$ and particle yields $Y$ were calculated for each centrality class.

In figure 1 the NA57 yields of hyperons from central $\mathrm{Pb}-\mathrm{Pb}$ interactions at 40 and $158 \mathrm{~A} \mathrm{GeV} / \mathrm{c}$ are presented and compared with those obtained by the STAR collaboration in $\sqrt{s_{N N}}=130 \mathrm{GeV} \mathrm{Au}-\mathrm{Au}$ collisions at RHIC [ 8, 9, 10. In order to compare data close in centrality with those of STAR $(5 \%, 10 \%$ and $11 \%$ most central collisions for $\Lambda, \Xi$ and $\Omega$ ) we have selected NA57 data for $5 \%$ most central events for $\Lambda$ and $11 \%$ most central events for $\Xi$ and $\Omega$. The $\Lambda$ and $\Xi^{-}$yields are almost constant in the full considered energy range, the $\Omega^{-}$yield increases with the energy. All anti-hyperon yields show a steep energy dependence.
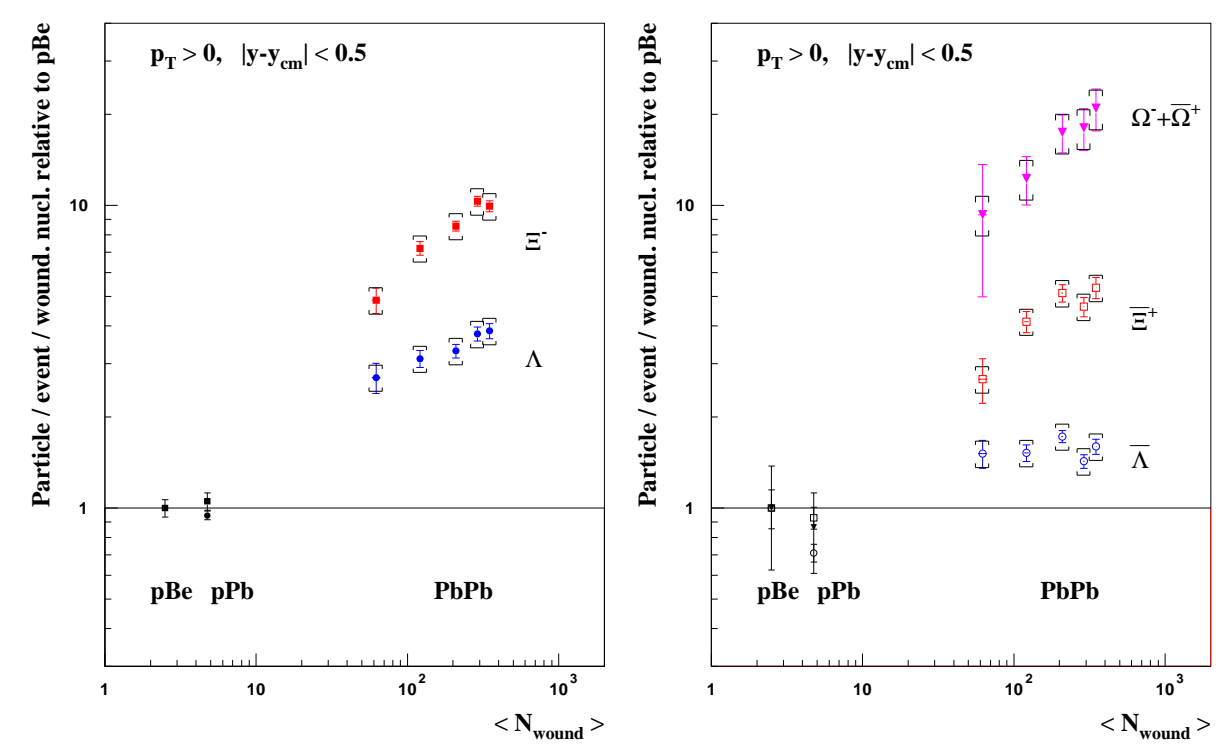

Fig. 2. Hyperon enhancements $E$ at $158 A \mathrm{GeV} / c$ as a function of the number of wounded nucleons. The symbol $\square$ indicates the systematic error.

Let us turn now to strangeness enhancements. Having measured the midrapidity particle yields $Y$ for both $\mathrm{Pb}-\mathrm{Pb}$ and $\mathrm{p}-\mathrm{Be}$ collisions, one can determine the strangeness enhancement

\footnotetext{
${ }^{1}$ The centrality classes (1-4) correspond to the four classes used in the WA97 analysis, while the most peripheral class 0 (with $\left\langle N_{\text {wound }}\right\rangle=58 \pm 4$ ) is accessible to NA57 only. The class 4 corresponds to the $5 \%$ and classes $3+4$ to the $11 \%$ most central fractions of events respectively.
} 


$$
E=\left(\frac{Y}{\left\langle N_{\text {wound }}\right\rangle}\right)_{\mathrm{Pb}-\mathrm{Pb}} /\left(\frac{Y}{\left\langle N_{\text {wound }}\right\rangle}\right)_{\mathrm{p}-\mathrm{Be}} .
$$

The NA57 results on strangeness enhancements at $158 \mathrm{~A} \mathrm{GeV} / c$ are shown in fig. 2]

The enhancements are shown separately for particles containing at least one valence quark in common with the nucleon (left) and for those with no valence quark in common with the nucleon (right). Our results confirm the pattern of strangeness enhancements observed by the WA97 experiment [2]. The enhancement increases with the strangeness content of the hyperon. The $\mathrm{Pb}-\mathrm{Pb}$ data exhibit a significant centrality dependence of the yields per wounded nucleon for all hyperons except for $\bar{\Lambda}$. However, for the two most central classes 3 and $4(\simeq 10 \%$ most central fraction of collisions) a saturation of the enhancements is not excluded ${ }^{2}$.

\section{Transverse mass spectra}

The transverse mass distributions $1 / m_{\mathrm{T}} \mathrm{d} N / \mathrm{d} m_{\mathrm{T}}$ for hyperons and antihyperons from $\mathrm{Pb}-\mathrm{Pb}$ collisions at $158 \mathrm{AGeV} / c$ measured in the whole centrality range accessible to the experiment are shown in figure 3
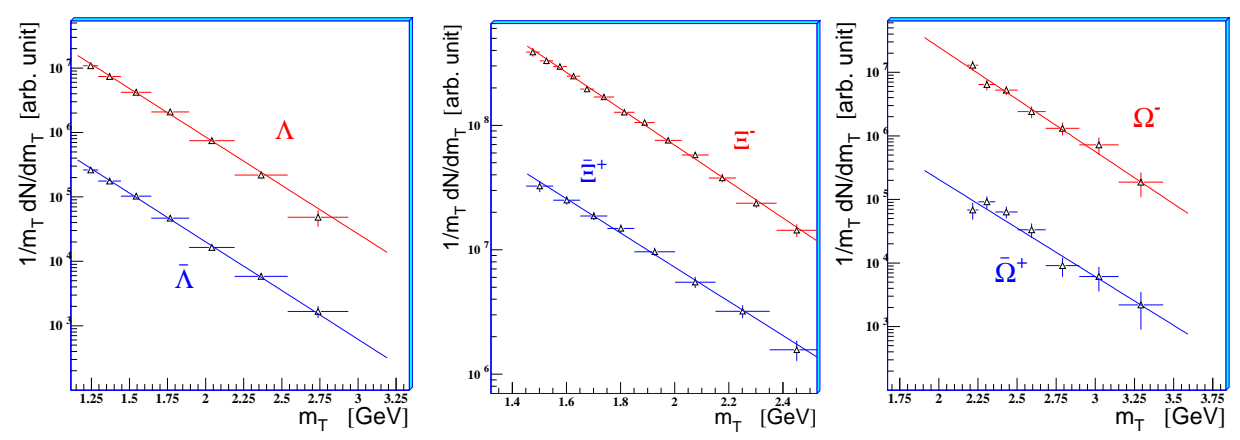

Fig. 3. Transverse mass spectra of hyperons from $158 A \mathrm{GeV} / c \mathrm{~Pb}-\mathrm{Pb}$ collisions.

The inverse slopes of the superimposed exponential functions correspond to the $T_{a p p}$ ('apparent temperature') values extracted from the maximum likelihood fit of equation (11) to the data. The shapes of all spectra are close to exponential functions with similar $T_{a p p}$ values $(\sim 300 \mathrm{MeV})$ for all hyperons and antihyperons ${ }^{3}$. The observed systematics of inverse slopes $T_{a p p}$ is usually interpreted as due to the combined contributions of thermal motion in the fireball and collective transverse flow.

\footnotetext{
${ }^{2}$ At the time of this manuscript's preparation the yields of $\Lambda, \bar{\Lambda}$ and $\Xi^{-}$hyperons from p-Be at $40 \mathrm{~A} \mathrm{GeV} / \mathrm{c}$ have been obtained and strangeness enhancements in $\mathrm{Pb}-\mathrm{Pb}$ data presented for the first time [11]. The strangeness enhancement pattern at $40 \mathrm{~A} \mathrm{GeV} / c$ exhibits similar properties and follows the same hierarchy as observed at $158 \mathrm{~A} \mathrm{GeV} / c$.

${ }^{3}$ The inverse slope values $T_{a p p}$ are in agreement within the errors with those measured over a narrower centrality range by the WA97 experiment [ 12 .
} 
For a more complex analysis aiming at disentangling the radial flow velocity $\beta_{\perp}(r)$ and the thermal freezout temperature $T$ we have utilized a model based on thermalization and a hydrodynamical description of transverse flow [13]. In this approach (the blast-wave model) the $m_{\mathrm{T}}$ distribution of hadron $i$ can be approximated as follows:

$$
\frac{\mathrm{d} N_{i}}{m_{\mathrm{T}} \mathrm{d} m_{\mathrm{T}}} \propto m_{\mathrm{T}} \int_{0}^{R} r \mathrm{~d} r K_{1}\left(\frac{m_{\mathrm{T}} \cosh \rho}{T}\right) I_{0}\left(\frac{p_{\mathrm{T}} \sinh \rho}{T}\right),
$$

where $R$ is the transverse system size, $K_{1}$ and $I_{0}$ are modified Bessel functions and $\rho=\operatorname{atanh} \beta_{\perp}(r)$ is a transverse boost. The model assumes kinetic freeze-out of matter at constant temperature $T$ and a cylindrically symmetric and longitudinally boost invariant expansion. The transverse velocity field is parametrised according to a power law

$$
\beta_{\perp}(r)=\beta_{s}\left(\frac{r}{R}\right)^{n} .
$$

Assuming a uniform particle density the average transverse velocity is related to the surface velocity $\beta_{s}$ by the formula $\left\langle\beta_{\perp}\right\rangle=2 /(2+n) \times \beta_{s}$.

Results of global fitting of the blast-wave model to all strange particle spectra measured by the NA57 experiment using velocity profiles with $n=0,1 / 2$ and 1 give similar freeze-out temperatures and average transverse velocities with adequate statistical significance of fits. In the following, we report the results for a linear $(n=1)$ velocity profile.

As shown in the left portion of figure 4 it is possible to obtain a successful description of all particle spectra with common values of the thermal freeze-out
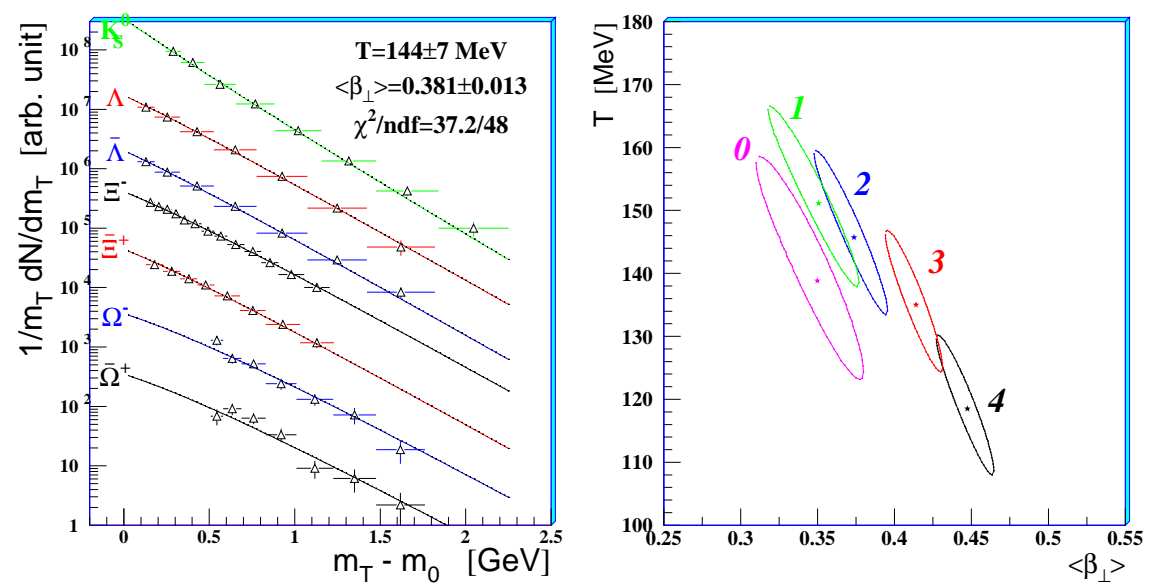

Fig. 4. Result of simultaneous fitting of equation (4) to the hyperon $m_{\mathrm{T}}$ spectra measured in the whole NA57 centrality range. Preliminary $\mathrm{K}_{\mathrm{S}}^{0}$ data are also included (left). The $1 \sigma$ confidence level contours for blast-wave fits to the spectra corresponding to individual centrality classes (right). 
temperature $T$ and average transverse flow velocity $\left\langle\beta_{\perp}\right\rangle$. The fitted values of these quantities and their statistical errors are also shown in figure $4^{4}$.

In order to check whether some particle species deviate from the common freezeout description determined according to this model, we have performed separate fitting of the $m_{\mathrm{T}}$ spectra for singly strange and multi-strange particles (for details see [11, 14]). Results of this analysis show that $\Xi$ hyperons undergo a thermal freeze-out which is compatible with that of $\mathrm{K}_{S}^{0}$ and $\Lambda$. The spectrum of $\Omega$ hyperons, although with limited statistics, indicates a different behaviour compatible with possible earlier freeze-out.

In order to investigate the centrality dependence of the freeze-out parameters we have also performed the global fits of $m_{\mathrm{T}}$ spectra for each of the five NA57 centrality classes. The results of these fits are summarized in the right portion of figure 4 Apart from the most peripheral interactions, the centrality dependence of the $T$ and $\left\langle\beta_{\perp}\right\rangle$ parameters show opposite trends: the freeze-out temperature is decreasing and the average flow velocity is increasing with increasing centrality of collision.

\section{Conclusions}

The NA57 data on the normalized hyperon yields $E$ at $158 A \mathrm{GeV} / c$ confirm the pattern of strangeness enhancements found by WA97: the enhancement increases with the strangeness content of the particle reaching a factor $\simeq 20$ for the triplystrange $\Omega$ hyperons. A significant centrality dependence of the enhancement for all hyperons and antihyperons (except for the $\bar{\Lambda}$ ) is observed.

The analysis of the transverse mass spectra in the framework of the blast-wave hydrodynamically inspired model shows that $m_{\mathrm{T}}$ spectra for all the particles under study can be successfully described by common values of the freeze-out temperature and average transverse velocity. However, the inverse slope of the $\Omega$ hyperon deviates from the prediction of the blast-wave model tuned to high statistics spectra of lighter strange particles. A centrality dependence of the freeze-out parameters $T$ and $\left\langle\beta_{\perp}\right\rangle$ is observed.

\section{References}

1. Proceedings of 7th International Conference on Strangeness in Quark Matter, eds. S.A. Bass, B. Müller, G.S.F. Stephans and T. Ullrich, J. Phys. G: Nucl. Part. Phys. 30 (2004) Number 1.

2. E. Andersen et al., Phys. Lett. B449 (1999) 401, F. Antinori et al., Nucl. Phys. A661 (1999) 130c.

3. J. Rafelski and B. Müller, Phys. Rev. Lett. 48 (1982) 1066; Phys. Rev. Lett. 56 (1986) 2334.

\footnotetext{
${ }^{4}$ The systematic errors of quantities $T$ and $\left\langle\beta_{\perp}\right\rangle$ are estimated to be $\pm 14 \mathrm{MeV}$ and \pm 0.012 respectively.
} 
4. V. Manzari et al., J. Phys. G: Nucl. Part. Phys. 25 (1999) 473.

5. V. Manzari et al., Nucl. Phys. A661 (1999) 716c.

6. V. Manzari et al., Nucl. Phys. A715 (2003) 140c.

7. N. Carrer et al., J. Phys. G: Nucl. Part. Phys. 27 (2001) 391.

8. C. Adler et al., Phys. Rev. Lett. 89 (2002) 092301.

9. J. Castillo et al., Nucl. Phys. A715 (2003) 518c.

10. C. Suire et al., Nucl. Phys. A715 (2003) 470c.

11. G.E. Bruno et al., Plenary NA57 talk at the Quark Matter 2004 conference (Oakland, California, 11-17 Jan. 2004), to be published in J. Phys. G: Nucl. Part. Phys.

12. F. Antinori et al., Eur. Phys. J. C14 (2000) 633.

13. E. Schnedermann, J. Sollfrank and U. Heinz. Phys. Rev. C48 (1993) 2462.

14. F. Antinori et al., J. Phys. G: Nucl. Part. Phys. 30 (2004) 823. 